\title{
Minute perforation after argon plasma coagulation for management of small colonic polyps
}

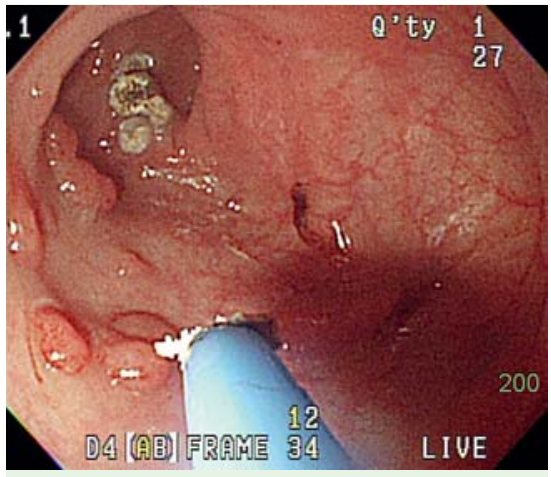

Fig. 1 Most of the small polyps in the colon were ablated using argon plasma coagulation (APC).

A 53-year-old woman presented with a 6month history of chronic constipation and abdominal bloating. Because of concern about a colorectal lesion, colonoscopy was done and hundreds of small polyps were found throughout the colon, most being less than $4 \mathrm{~mm}$ in diameter. Some of the bigger polyps were removed by polypectomy and were found at pathologic examination to be tubular adenoma. As the patient was unwilling to undergo a rectum-preserving total colectomy, argon plasma coagulation (APC) was selected to ablate the remaining polyps as far as possible ( $\bullet$ Fig. 1).

APC was done using an Olympus system (PSD-60; Olympus, Tokyo, Japan) at settings of argon flow rate $1 \mathrm{l} /$ minute, power $40 \mathrm{~W}$, effect 1.

The patient tolerated the 1-hour procedure well until the end, when severe abdominal distension without rebound tenderness was noted. Because of the persistent abdominal discomfort, a standing chest radiograph was obtained which showed massive free air in the peritoneum ( $\bullet$ Fig. 2).

Laparotomy was done immediately. The entire colon was checked carefully and a tiny perforation was found at the transverse colon ( Fig. 3, arrow).

After surgical closure of the perforation, the patient recovered uneventfully and was discharged 2 weeks later.

APC is safe and effective for the management of gastrointestinal tract bleeding, polyp remnants, and watermelon stom-

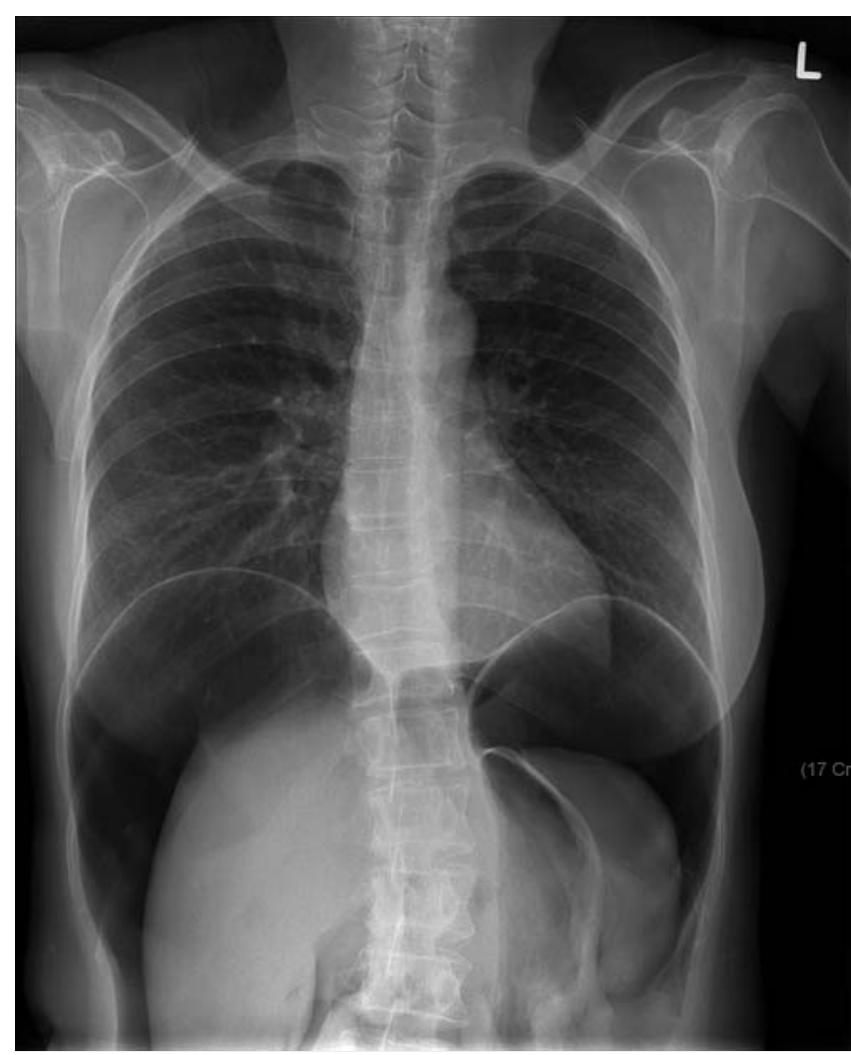

Fig. 2 At 3 hours after colonoscopy, a standing chest radiograph revealed massive pneumoperitoneum.

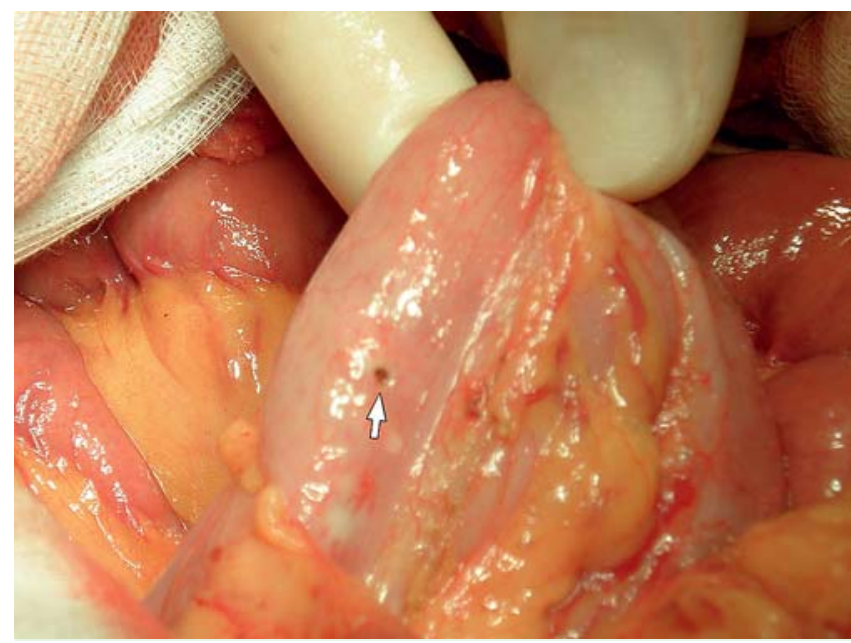

Fig. 3 At laparotomy, a tiny perforation (arrow) was found at the transverse colon.

ach, ablation of precancerous lesions, and so on [1 - 3]. An asymptomatic air accumulation in the peritoneum may develop after APC because the high argon flow induces submucosal emphysema with a leakage of gas through the gastrointestinal tract wall. Conservative treatment is suggested for this situation [4]. As our patient underwent APC ablation of numer- ous colonic polyps, the air insufflation was prolonged, leading to severe pneumoperitoneum even though there was only a tiny perforation. Thus, it is very important to distinguish between the symptomatic 'perforation' and the asymptomatic 'air accumulation' as causes of pneumoperitoneum. 


\section{Endoscopy_UCTN_Code_CPL_1AJ_2AI}

C.-H. Chuang ${ }^{1}$, T.-C. Chou' ${ }^{2}$, C.-Y. Chen ${ }^{1}$ ${ }^{1}$ Department of Internal Medicine, Medical College and Hospital, National Cheng Kung University, Tainan, Taiwan

2 Department of Surgery, Medical College and Hospital, National Cheng Kung University, Tainan, Taiwan

\section{References}

1 Manner H, May A, Faerber M et al. Safety and efficacy of a new high power argon plasma coagulation system (hp-APC) in lesions of the upper gastrointestinal tract. Dig Liver Dis 2006; 38: $471-478$

2 Izquierdo S, Rey E, Gutierrez Del Olmo A et al. Polyp as a complication of argon plasma coagulation in watermelon stomach. Endoscopy 2005; 37: 921

3 Neneman B, Gasiorowska A, Malecka-Panas $E$. The efficacy and safety of argon plasma coagulation (APC) in the management of polyp remnants in stomach and colon. Adv Med Sci 2006; 51: 88-93

4 Manes G, Imbesi V, Bianchi-Porro G. Pneumoperitoneum after argon plasma coagulation treatment: perforation or accumulation of air in the cavity? Endoscopy 2007; 39 Suppl 1: E98

\section{Bibliography}

DOI 10.1055/s-0029-1214432

Endoscopy 2009; 41: E251 -E252

(c) Georg Thieme Verlag KG Stuttgart · New York . ISSN 0013-726X

\section{Corresponding author}

\section{C.-Y. Chen, MD}

Department of Internal Medicine National Cheng Kung University Hospital 138 Sheng-Li Road, Tainan

Taiwan

Fax: +886-6-2766116

chiungyu@mail.ncku.edu.tw 